\title{
Pontocerebellar hypoplasia type 2: a neuropathological update
}

\author{
Peter G. Barth • Eleonora Aronica • Linda de Vries • Peter G. J. Nikkels • \\ Wiep Scheper · Jeroen J. Hoozemans · Bwe - Tien Poll-The $\cdot$ Dirk Troost
}

Received: 17 May 2007 / Revised: 18 June 2007 / Accepted: 27 June 2007 / Published online: 20 July 2007

(C) Springer-Verlag 2007

\begin{abstract}
Pontocerebellar hypoplasia type 2 (PCH-2; MIM 277470), an autosomal recessive neurodegeneration with fetal onset, was studied in six autopsies with ages at death ranging between 1 and 22 years. Three patients were distantly related. A case of olivopontocerebellar hypoplasia (OPCH; MIM 225753) was studied for comparison. Typical findings are: short cerebellar folia with poor branching ("hypoplasia"), relative sparing of the vermis, sharply demarcated areas of full thickness loss of cerebellar cortex probably resulting from regression at an early stage of development, segmental loss of dentate nuclei with
\end{abstract}

P. G. Barth $(\bowtie) \cdot$ E. Aronica · D. Troost

Department of Neuropathology, Academic Medical Centre,

University of Amsterdam, P.O. Box 22700,

1100 DE Amsterdam, The Netherlands

e-mail: p.g.barth@amc.uva.nl

P. G. Barth - B. - T. Poll-The

Department of Pediatric Neurology,

Emma Children's Hospital/AMC, University of Amsterdam,

P.O. Box 22700, 1100 DE Amsterdam, The Netherlands

L. de Vries

Department of Neonatology, Wilhelmina Children's Hospital,

University Medical Centre, Utrecht, The Netherlands

P. G. J. Nikkels

Department of Pathology,

University Medical Centre, Utrecht, The Netherlands

W. Scheper

Neurogenetics Laboratory/Department of Neurology, Academic Medical Centre, University of Amsterdam, Amsterdam, The Netherlands

J. J. Hoozemans

Department of Pathology, VU Medical Centre,

Free University, Amsterdam, The Netherlands preserved islands and reactive changes, segmental loss in the inferior olivary nucleus with reactive changes, loss of ventral pontine nuclei with near absence of transverse pontine fibers and sparing of spinal anterior horn cells. Variable findings are: cystic cerebellar degeneration, found in two, with vascular changes limited to the cerebellum in one. Comparison to olivopontocerebellar hypoplasia $(\mathrm{OPCH})$ strongly suggests a continuum of pathology between this disorder and $\mathrm{PCH}-2$. Immunohistochemical evaluation of the endoplasmic reticulum stress response is negative. We conclude that the neuropathological findings in $\mathrm{PCH}-2$ are sufficiently specific to enable an unequivocal diagnosis based on neuropathology.

Keywords Cerebellum · Pontocerebellar hypoplasia . Olivary nucleus $\cdot$ Pons

\section{Introduction}

The term pontocerebellar hypoplasia $(\mathrm{PCH})$ is applied to a group of autosomal recessively inherited neurodegenerations with prenatal onset. The distinguishing attribute of this group is the presence of combined pontocerebellar hypoplasia and atrophy present at the time of birth.

Supratentorial structures are affected, though less prominently. The group has not been clarified genetically or biochemically. An initial proposal for classification [6] is based on two types in which type 1 ( $\mathrm{PCH}-1)$ associates PCH with spinal anterior horn cell degeneration [14, 33], while type 2 ( $\mathrm{PCH}-2)$ associates $\mathrm{PCH}$ with clinical extrapyramidal involvement and absence of anterior horn degeneration [5-7, 37, 44]. Early detailed neuropathological reports on PCH $[9,10,26]$ in retrospect may have been cases of PCH-2, but cannot be matched with the customary 
classification because of lack of clinical details. Neuropathological finding in $\mathrm{PCH}-2[5,7,37,41]$ is microencephaly with severe cerebellar and ventral pontine hypoplasia. Typical pathological findings are: (1) subtotal loss of ventral pontine neurons and transverse pontine fibers with preservation of long fiber tracts; (2) atrophy and hypoplasia of the cerebellar hemispheres with relative sparing of the vermis and flocculi, variable loss of Purkinje cells and internal granule cells and reduction in folial length; (3) patchy loss of cerebellar dentate neurons, with the remaining dentate neurons grouped in "islands"; (4) loss of neurons in the inferior olivary nucleus with the winding pattern essentially spared; (5) absence of the medullary arcuate nuclei. Findings in PCH-1 are essentially similar, but preservation of spinal anterior horn cells distinguishes PCH-2 from PCH-1 [14]. Microscopic supratentorial findings in $\mathrm{PCH}-2$ are non-specific with neuronal loss in various compartments, including the cerebral cortex, while myelin and myelination remain unaffected. Ultrastructural findings in a single reported neocortical biopsy are progressive neuronal loss in all layers and a peculiar degeneration in neurons in all layers that appear to start with patches of darkened endoplasmic reticulum [5]. Diagnosis during life is based on a combination of findings on MRI (Fig. 1), a profile of clinical neurological deficits that includes severe cognitive delay, swallowing disturbance and chorea/dystonia (less often, spasticity), exclusion of metabolic and chromosomal disorders with a similar MRI pattern and a family history compatible with autosomal recessive inheritance. In typical cases, behavioral and motor development is almost stagnant from the beginning, with onset of chorea/dystonia during the first year and progressive microcephaly [7, 44]. Differential diagnosis requires exclusion of glycosylation disorders, especially congenital disorder of glycosylation type 1A (CDG1A) [2, 21, 23] and Muscle-Eye-Brain disease [16, 29], sequelae of extreme prematurity [28] and chromosomal disorders [4]. Overlapping neuropathological features exist between PCH-2 and olivopontocerebellar hypoplasia (OPCH), a more severe disorder with an essentially similar combination of hypoplasia and degeneration affecting the hindbrain $[3,11,18,35]$. Autosomal recessive inheritance in $\mathrm{OPCH}$ is suggested by its recurrence pattern in families. Clinical findings are variable, but include such indicators of prenatal onset as polyhydramnios and contractures. Its main structural features are severe cerebellar hypoplasia with emphasis on the hemispheres, absence of neurons in the ventral pons, subtotal absence of cerebellar dentate nuclei, diminished or absent olivary winding and absence of spinal anterior horn involvement. In a recent PCH classification, the original types 1 and 2 have remained identical, while olivopontocerebellar hypoplasia has become type 4, $\mathrm{PCH}-4$ [36]. Type 3, with optic atrophy as its main distinguishing clinical feature [38], has not been studied neuropathologically yet, while type 5 until now is represented by a single family. The number of papers addressing the neuropathology of PCH-2 with sufficient clinical detail to support a type 2 classification is limited [5, 37, 41] and results of immunohistochemistry have not been reported yet. The aim of the present report is to provide a range of pathological findings based on a series of six autopsies with ages at death varying between 15 months and 22 years and to provide an update on neurodegenerative features using more specific staining techniques. Special attention is given to variability in cerebellar degeneration, degenerative changes in pons and lower brainstem and the types of astroglial and microglial reaction. Three patients (no. 4, 5, 6) originate from an area with a high degree of intermarriage and are related by genealogy. A case of olivopontocerebellar hypoplasia (PCH-4) is included for comparison of its neuropathology to PCH-2.

\section{Materials and methods}

Standard autopsies were performed on all patients mentioned except patient 1 who underwent a coroner's autopsy with only the cerebellum and brainstem made available for the present study (Table 1).
Fig. 1 MRI of patient 6 at 2 months. a Midsagittal inversion recovery sequence: flat aspect of ventral pons and moderate vermal hypoplasia. b Coronal $\mathrm{T} 2$-weighted image: severe hypoplasia of the cerebellar hemispheres (arrows) together filling only a fraction of the posterior fossa
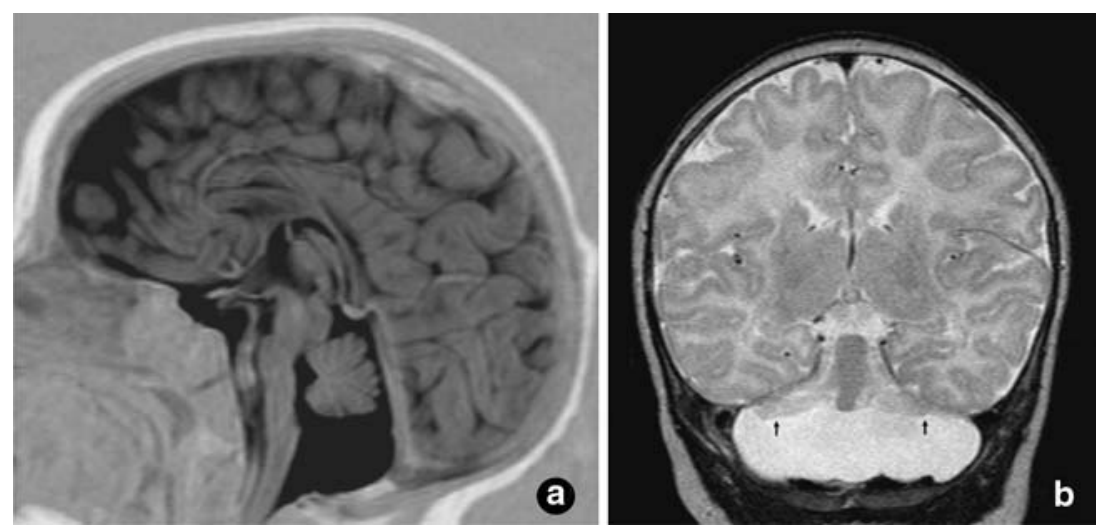


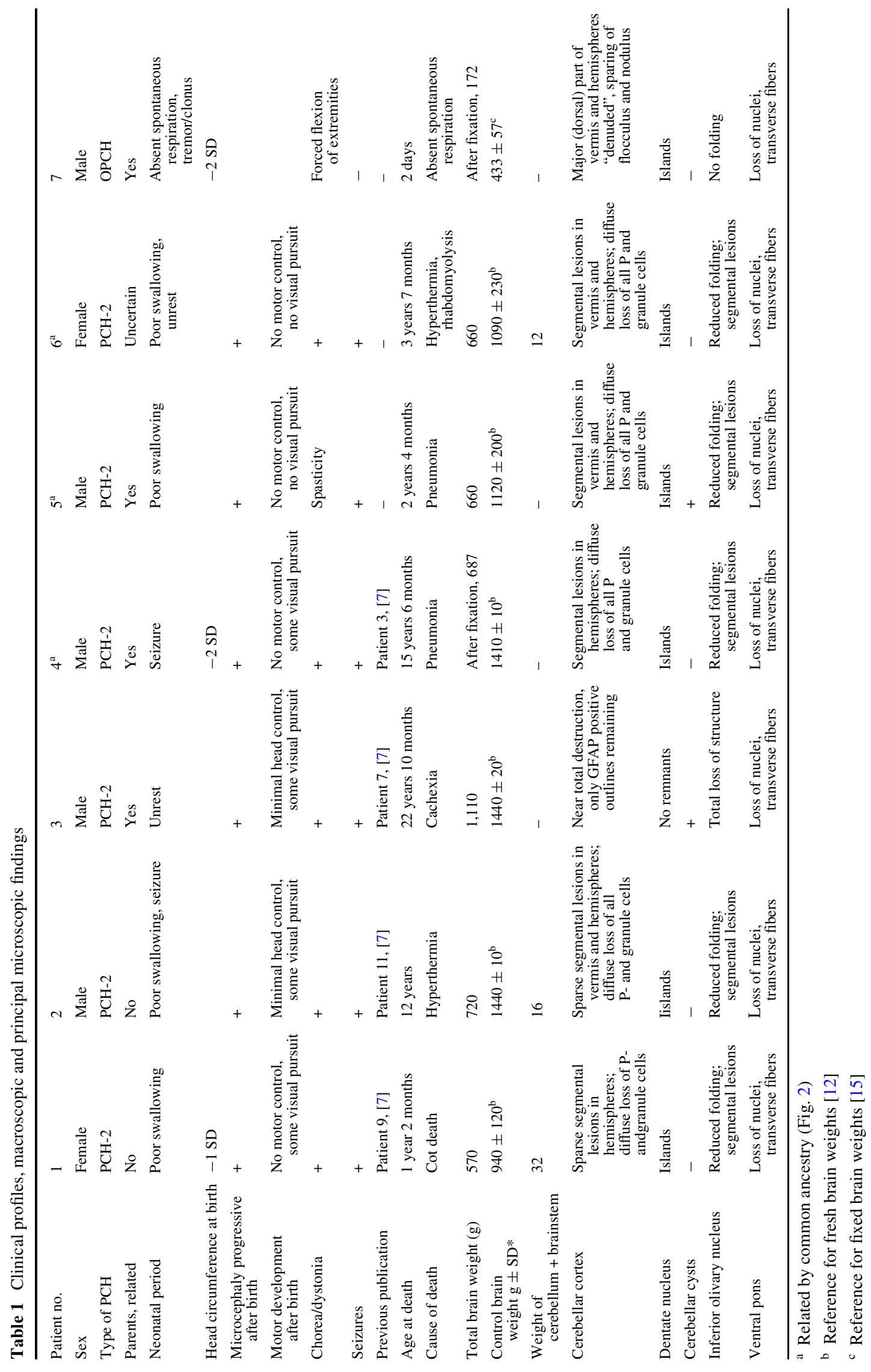




\section{Tissue preparation and immunocytochemistry}

Formalin-fixed, paraffin-embedded tissue was sectioned at $6 \mu \mathrm{m}$ and mounted on organosilane-coated slides (SIGMA, St Louis, MO, USA). Representative sections of all specimens were processed for immunocytochemical reactions to synaptophysin (polyclonal rabbit, DAKO, 1:200), microtubule-associated protein (MAP2; polyclonal rabbit, Sigma, 1:2,000), calbindin (mouse clone CL-300, Sigma, 1:400), SMI31 (Sternberger Monocl., IgG 1, 1:20,000), phosphorylated neurofilament (NF; mAb clone 2F11, DAKO, 1:500), myelin basic proteïn (MBP; polyclonal, Neomarkers/RB1460-A, 1:400), vimentin (mouse clone V9, DAKO, Denmark, 1:1,000), glial fibrillary acidic protein (GFAP; polyclonal rabbit, DAKO, Denmark, 1:4,000), (HLA)-DP, DQ, DR (mouse clone CR3/43, DAKO Glostrup), HLADR (mouse clone Tal1b5, Sigma, USA, 1:200), CD68 (KP1 clone, Novocastra), caspase-3 (polyclonal, 1:100 dilution, Cell Signalling Technology, Beverly, MA, USA), ubiquitine (polyclonal, DAKO/Z458, 1:1,600), $\alpha$-synuclein (polyclonal, Affiniti/SA 3400, 1:2,000), BiP/GrP/78 [polyclonal rabbit, 1:100 dilution Santa Cruz Biotechnology (SCBT), Santa Cruz, CA, USA], phosphorylated (p)PERK (pThr981, polyclonal rabbit, 1:800 dilution, SCBT) and phosphorylated (p)eIF2 $\alpha$ (pSer51, polyclonal rabbit, 1:500 dilution, Sigma).

Paraffin sections were routinely stained with HE, LFB and Nissl. Cerebellar sections were silver stained according to Bielschowsky. Immunocytochemistry was carried out on paraffin-embedded tissue as previously described [2, 19]. The sections were incubated for $1 \mathrm{~h}$ at room temperature followed by incubation at $4{ }^{\circ} \mathrm{C}$ overnight with primary antibodies. Single-label immunocytochemistry was performed using avidin-biotin peroxidase method. Chromogen 3,3diaminobenzidine or 3-amino-9-ethyl-carbazole (AEC, Zymed, San Francisco, CA) was used and nuclei were stained with hematoxylin. Sections incubated without the primary $\mathrm{Ab}$, with preimmune sera were essentially blank. For double labeling (with ubiquitin and HLA-DR), sections were incubated for $2 \mathrm{~h}$ at RT with Alexa Fluor ${ }^{\circledR} 568$ and Alexa Fluor ${ }^{\circledR} 488$ (anti-rabbit IgG, anti-mouse IgG; Molecular probes, Eugene, USA). The sections were then analyzed by means of a laser scanning confocal microscope (Bio-Rad, Hercules, CA, USA; MRC1024) equipped with an argon-ion laser.

\section{Results}

Details of each patient are given in Table 1. Figure 2 shows the pedigree, which links patients 4,5 and 6 to a common ancestry. Parents of patients 4 and 5 are consanguineous. The three parental couples originate from the Dutch

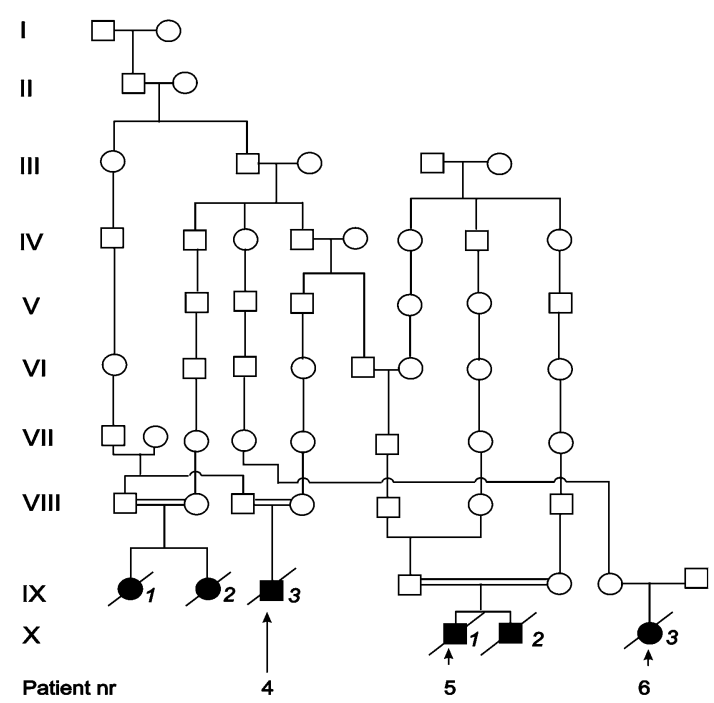

Fig. 2 Pedigree linking patients 4, 5 and 6 in the present report; IX, 1 and IX, 3 were reported before [5]

community in Volendam, which has a high degree of consanguinity. They link genetically to one or two ancestral couples: I, $1 \times$ I, 2 and III, $4 \times$ III, 5 born in the eighteenth century. A genealogical link between these ancestral couples, who carry identical family names, is suggested but yet unproven.

\section{Cerebellar cortex}

The cerebellar hemispheres are severely affected in all. On macroscopic examination, all major lobes are reduced in width and dorsoventrally flattened. Size was best preserved in the transverse plane. This asymmetric diminution in size and the relative sparing of the vermis lend the cerebella a butterfly or batwing aspect (Fig. 1b). Four of the six cerebella (cases 2, 4-6) are sectioned perpendicular to the folia with the line of the sectioning running from the culmen to the posterior pole. These sections include the flocculus. In two cases $(1,3)$, transverse sections of hemispheres and vermis are prepared. Low magnification (Fig. 3) shows the diminished size of the cerebellar hemispheres, folial shortening and diminished number of folial branches compared to a control (Fig. 3a, b). In the most affected case (Fig. 3g, h), no branches are seen at all, while in a less affected case folial branches are reduced to two or less (Fig. 3e, f). The other cerebella displayed regular folial branching (Fig. 3c, d), though less in quantity than the control specimen (Fig. 3a, b).

The vermis is fully developed in all cases with the individual lobules well developed in size and branching pattern. Sagittal sections of the vermis, available in four, show some inequality in thickness of the white matter cores of the lobules, with the declive-folium-tuber complex the best 


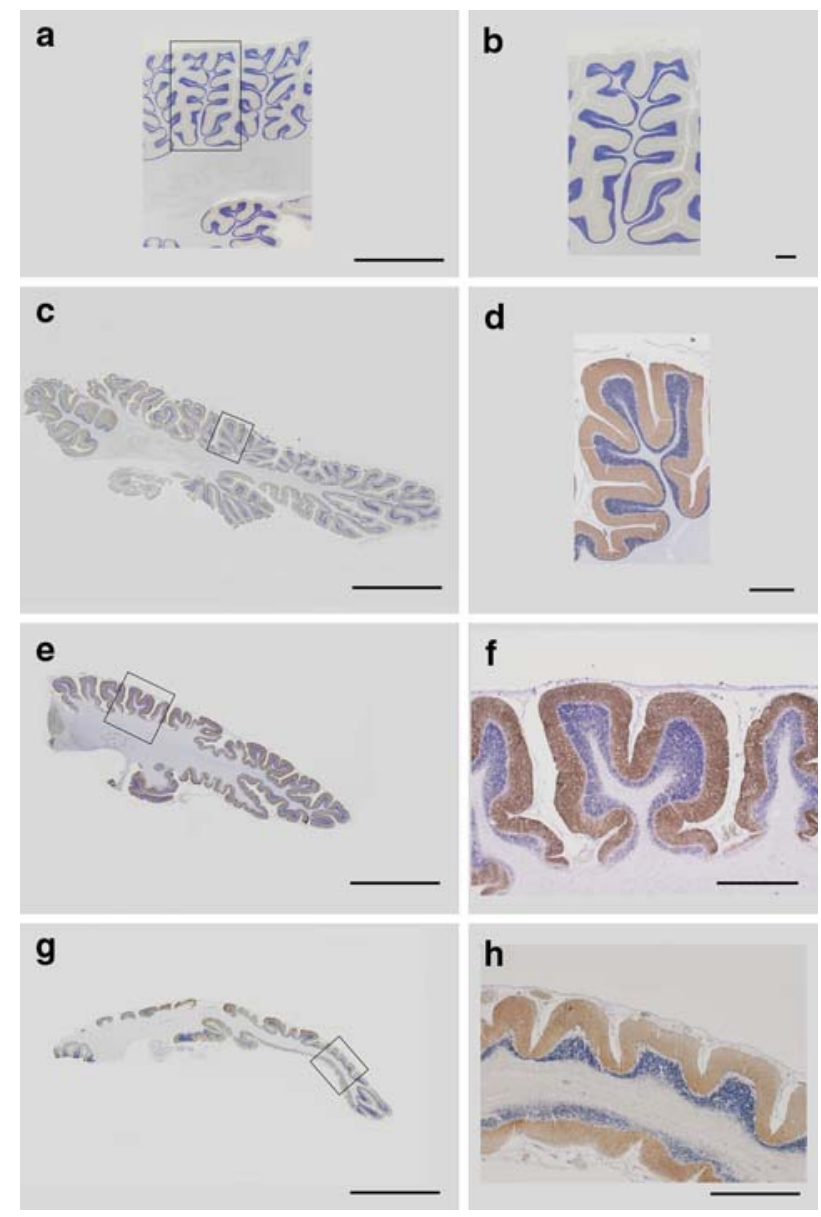

Fig. 3 Hypoplastic folia. Stains, synaptophysin. Sagittal sections of cerebellar hemispheres are seen on the left and detailed views on the right. Stunted folial growth with decreased number of folial branches. a, b Male, 8 years, with accidental death and normal findings on autopsy (control); c, $\mathbf{d}$ case $4 ; \mathbf{e}, \mathbf{f}$ case $6 ; \mathbf{g}, \mathbf{h}$ case 5 . Marker bars on the left $1 \mathrm{~cm}$, and on the right $1 \mathrm{~mm}$

preserved part in three (cases 2, 4, 5) and the pyramis in one (case 5; Fig. 4).

On microscopy, regressive changes are present in all, with the vermis relatively spared. In the vermis, diffuse but moderate loss of Purkinje cells, internal granule and folial white matter is seen in all lobules. Two cases present characteristic sharply delimited foci with full thickness loss of vermal cerebellar cortex with astroglial replacement $(2,5$; Fig. 4).

The hemispheric cerebellar cortex is affected in all cases by variable loss of Purkinje cells, thinning of the granule cell layer and inhomogeneous loss of myelinated fibers within folia and central white matter. The remaining axons are well myelinated and stain for SMI 131 and MBP. In addition to diffuse cortical changes, sharply delimited areas of up to $2 \mathrm{~mm}$ in width with loss of all cortical neurons are found in 5/6 cases $(1,2,4,5,6)$. These areas locate mostly, but not exclusively, to stretches of cortex connecting adjacent folia. The number of these areas varies between two per whole cerebellar hemispheric section $(1,2)$ to ten or more (5, 6; Fig. 5). In one case (3), representing the oldest surviving patient, destruction of the whole cerebellum is almost complete with subtotal loss of all neuronal elements (Fig. 10a). Even in this case, patches of whole thickness loss of cortex alternate with segments of cortex where gliosis still preserves the original frame of cortical structure (Fig. 10a). In all cases, the flocculus belongs to the relatively spared areas of the hemispheric cortex. In one case, the sparing of the flocculus stands out in sharp contrast to the rest of the hemispheric cortex (Fig. 6).

\section{Dentate nucleus}

The dentate nucleus is affected in all cases by major loss of dentate neurons and by residual cells being grouped in "islands" (Figs. 7, 8). "Islands" as well as individual neurons are surrounded by whorls of nerve fibers. Residual neurons and the proximal parts of their dendrites stain positive for MAP-2 and synaptophysin. Synaptophysin staining is abundant on neurons and proximal dendrites signifying retained network activity in surviving neurons. A peculiar vacuolization surrounds individual dentate neurons (Fig. 8a, b). A proportion of these vacuoles stain positive for calbindin, suggestive of swollen axon terminals (Fig. 8c, d). Cerebellar white matter surrounding the dentate nucleus on the inside and outside shows thickened axons with diameters up to $12 \mu \mathrm{m}$ (Fig. 8). The other internal cerebellar nuclei could not be identified with certainty.

Cystic degeneration of the cerebellum

In two cases, destruction resulted in cyst formation (cases 3,5 ). MR investigation shows cystic change in the cerebellar hemispheres of patient 5 at the age of 1 month (Fig. 9b ). On autopsy the cysts are collapsed, and can only be retrieved by serial sectioning. The border of the cyst is lined by reactive astrocytes and macrophages (Fig. 9a, c). In case 3, cyst formation compounds the subtotal loss of cortex, dentate nuclei and fiber tracts. A remarkable involvement of small sized vessels, not present in the other cases, consists of intimal proliferation and splitting of the elastica interna. This abnormality is only present in the cerebellum and its surrounding arachnoid vessels (Fig. 10). Electron microscopy fails to show storage material or enlarged lysosomes (not shown). Double labeling for ubiquitin and HLA-DR shows that ubiquitin is mainly present in the vessel walls, and only in minor part associated with macrophages (HLA-DR; Fig. 10c). Vascular abnormalities are absent in other parts of the brain and in internal organs, including the kidneys. 
Fig. 4 Vermis cerebelli, case 5. Stains, a HE, b GFAP, c neurofilament protein, $\mathbf{d}$ vimentin. a-c Adjacent sections with circumscript segments of loss of all layers and glial reaction. Arrows in (a) point to circumscript areas of lost cortex. d Sharply demarcated foci and replacing bands of astrocytes possibly modified Bergmann cells. Marker bar in (d) $=500 \mu \mathrm{m}$

Fig. 5 Cerebellar hemispheric cortex with segmental lesions. Stains, a, h synaptophysin, b-d vimentin, e, g, i GFAP, f MAP-2. Case 6: opposing arrows mark lesions with loss of all cortical layers (a) and glial replacement (b). c, d Case 5: segment of whole thickness loss of cortex is replaced by a single layer of evenly spaced astrocytes, their topography compatible with modified Bergmann cells. e, f, g Case 4: segmental loss of cortex with glial replacement marked by opposing arrows; in (f) higher magnification shows a group of remaining isolated granule cells. $\mathbf{h}, \mathbf{i}$ Case 2: segmental loss of cortex on top of a folium in moderately hypoplastic cortex. Marker bars: $\mathbf{a}-\mathbf{c}, \mathbf{e}, \mathbf{g}-\mathrm{i}=0.5 \mathrm{~mm}$, $\mathbf{d}=0.25 \mathrm{~mm}, \mathbf{f}=50 \mu \mathrm{m}$
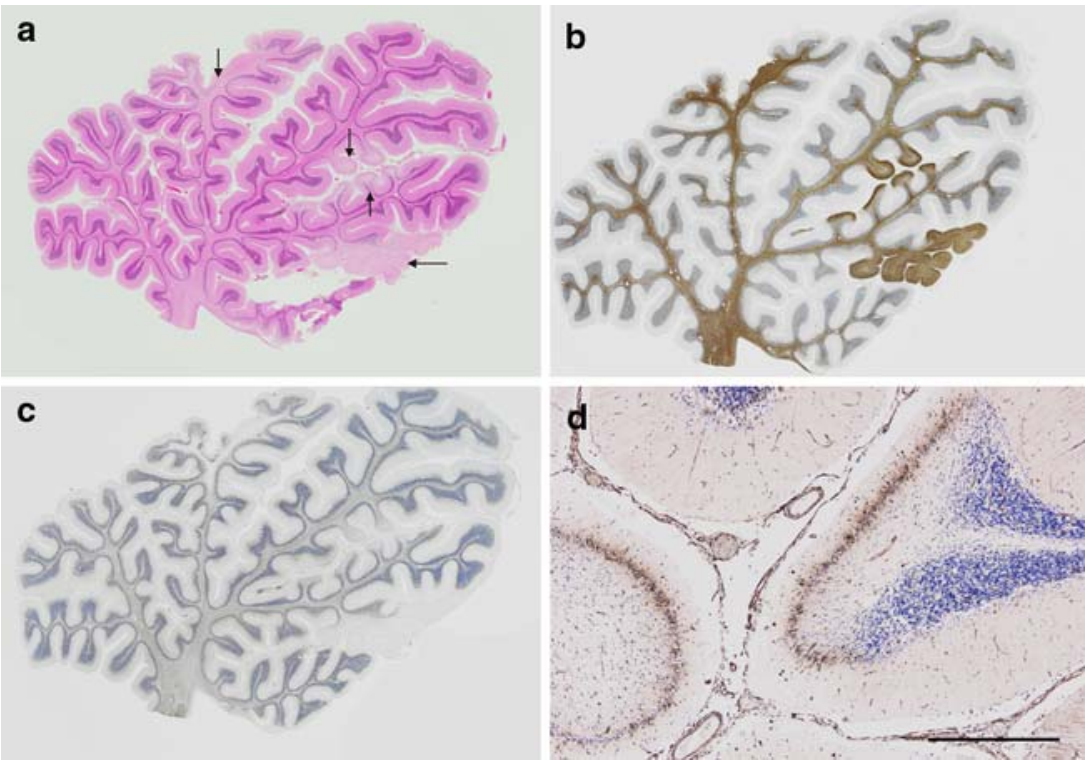
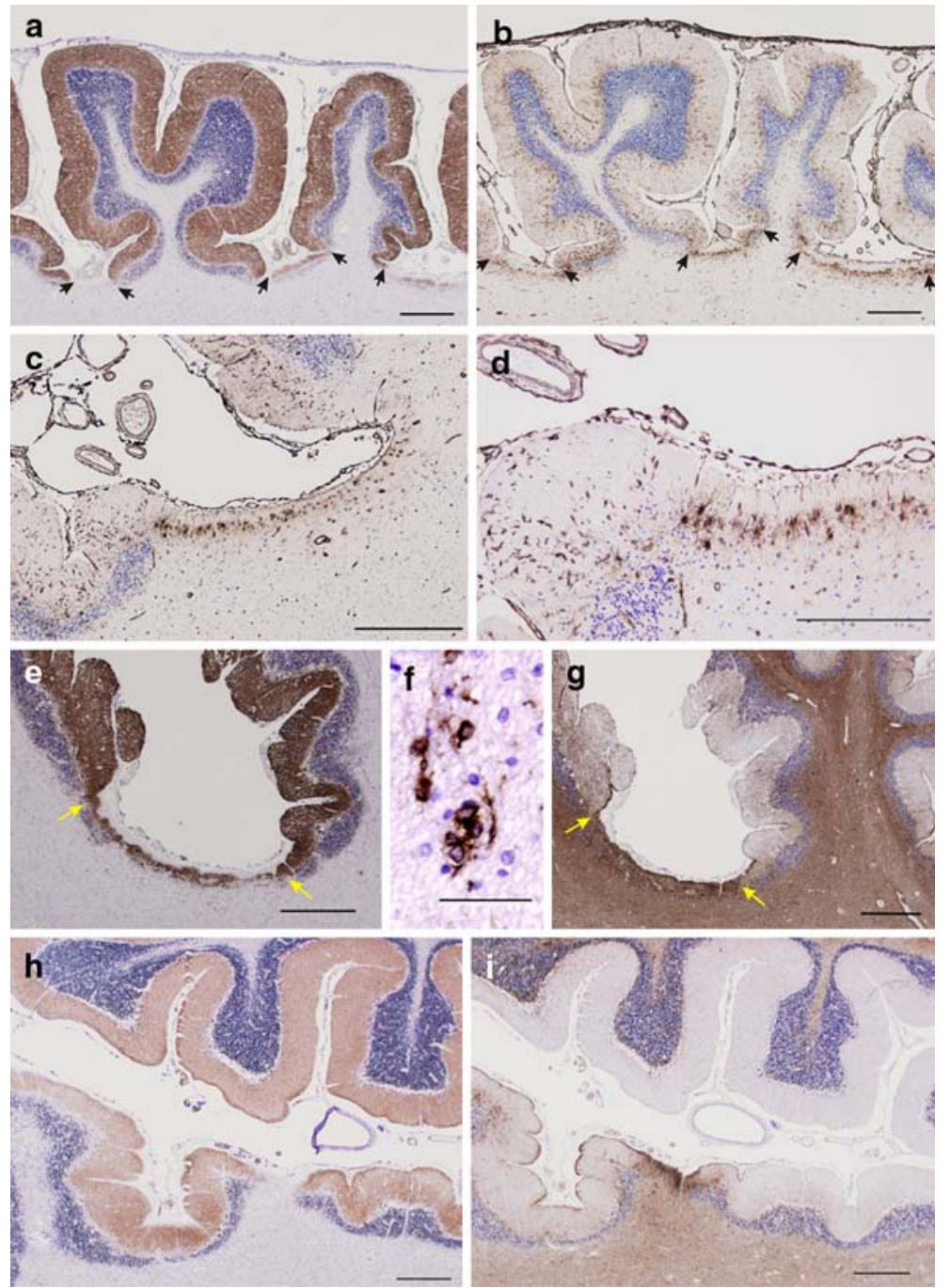


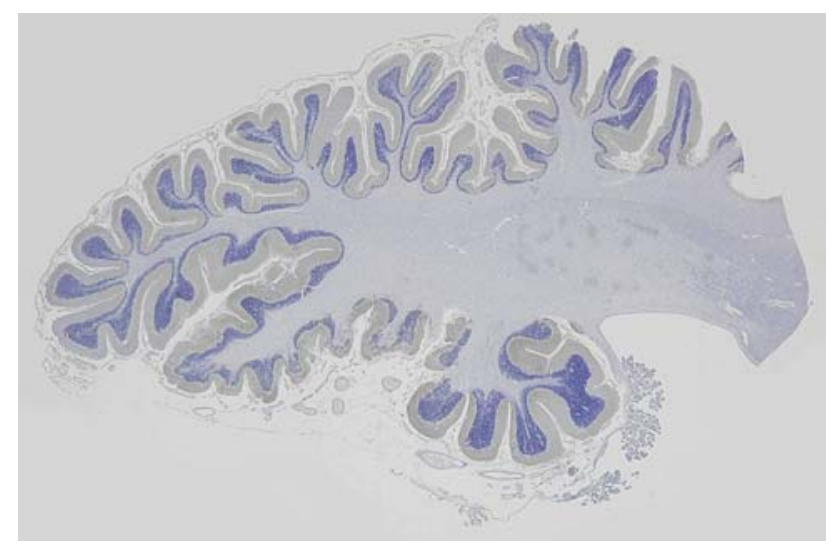

Fig. 6 Flocculus. Cerebellar hemispheric cortex, case 2, synaptophysin. Floccular cortex shows broader folial cores and denser granule cell population than other parts of the cortex

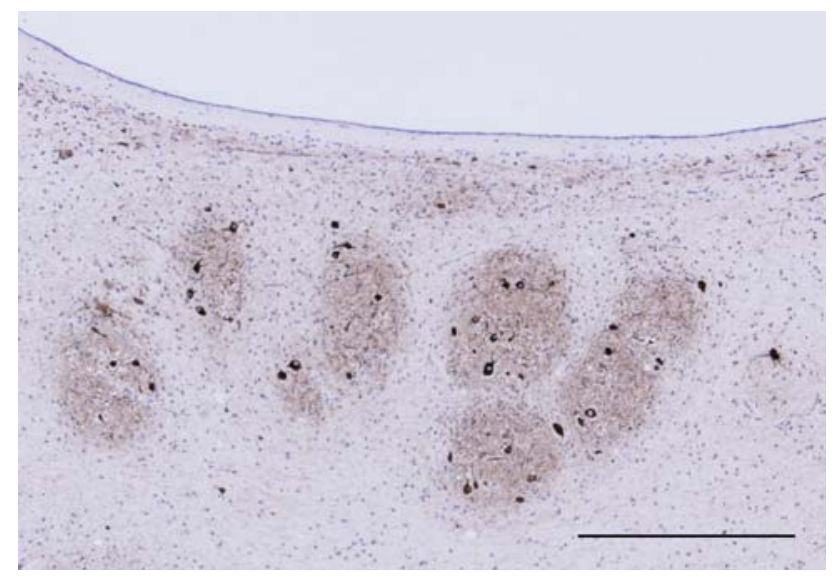

Fig. 7 Dentate nucleus, case 6. MAP-2. Loss of normal outline with remaining dentate neurons clustered in "islands". Marker bar $=1 \mathrm{~mm}$

\section{Inferior olivary nuclei}

The inferior olivary nucleus and the dorsal and medial accessory olivary nuclei are identified in each case. Mature folding of the inferior olivary nucleus is present in the lateral leaf and reduced in the medial leaf in one case (3; Fig. 11). In all others, folding is reduced in both leaves, the medial leaf being more affected than the lateral. Discontinuous loss of parts of the nerve cell band is noted in each case, best shown by MAP-2 staining (Fig. 11). In one case (3) nearly all olivary neurons, including the accessory olivary nuclei, are lost. Part of this loss is mirrored by vimentin positive astrocytes forming a ghost image of the lost neuronal framework (Fig. 11c, d). Macrophages are present within and around the vanished structure, but not in a topographically distinct pattern. In all cases, internal and external olivary fibers are reduced or lost entirely.

\section{Pons}

\section{Ventral pons}

Similar findings are present in all cases. Diminution in size of the pons to about half or less of its size in transverse diameter is entirely due to loss of its ventral compartment. The majority of ventral pontine neurons and transverse pontine fibers are lost with sparing of the long motor and sensory tracts. Prominent gliosis (Vim and GFA) and microglial activation are found in the affected region. Staining for synaptophysin and calbindin in the remaining neurons does not reveal specific patterns. Caspase and ubiquitin staining is negative in the few remaining neurons.

\section{Pontine tegmentum}

No abnormalities are encountered in the cranial nerve nuclei, raphe or central tegmental tract. Ubiquitin staining in the reticular nuclei, especially the nucleus raphes magnus in case 3 , reveals numerous neurons with ubiquitin-positive cytoplasmic inclusions. Caspase-3 staining is negative in all cases.

Other findings in the brainstem

Total absence of the medullary arcuate nuclei is found in all cases. Widespread presence of lipopigment (lipofuscin) is observed in all cases, especially in larger neurons (dentate nuclei, olivary nuclei, pontine neurons, brainstem tegmentum and cerebral cortex), even in the youngest individuals. Staining for $\alpha$-synuclein was negative in the brainstem as well as in the cerebellum in all cases.

Midbrain, thalamus and basal ganglia

Nucleus ruber has diminished size and cell density in case 3. This case contrasts with other cases by its subtotal destruction of the cerebellum. In all available cases (2-6), the caudate nuclei are macroscopically atrophic. There is a general increase of astrocytes and microglia throughout the basal ganglia and thalami. Myelination is normal. No specific abnormalities are found. In one case severe necrotic changes are found in the globus pallidus, representing acute postischemic damage.

\section{Cerebral cortex}

Macroscopic gyral atrophy is seen in three of five cortices available for study. The temporal lobes are affected in one (case 2), the frontal and temporal lobes in one (case 3) and all lobes in one (case 4). Myelination is normal in large sections (luxol fast blue), even in atrophic parts. Cortical 
Fig. 8 Dentate nucleus, details. Stains: a MAP-2, b MBP, c, d calbindin, e synaptophysin, f SMI31. a Case 6: fine vacuolation surrounding neurons. b Case 1: myelinated fibers separate individual neurons and their surrounding vacuoles. c, d Cases 6, 1: some vacuoles are decorated by antibody; e Case 2: concentration of vesicles (dots) indicates high synaptic density on soma and dendrites of surviving neuron. $\mathbf{f}$ Case 1 : thickened axons in hilus of dentate nucleus. Marker bars $=100 \mu \mathrm{m}$
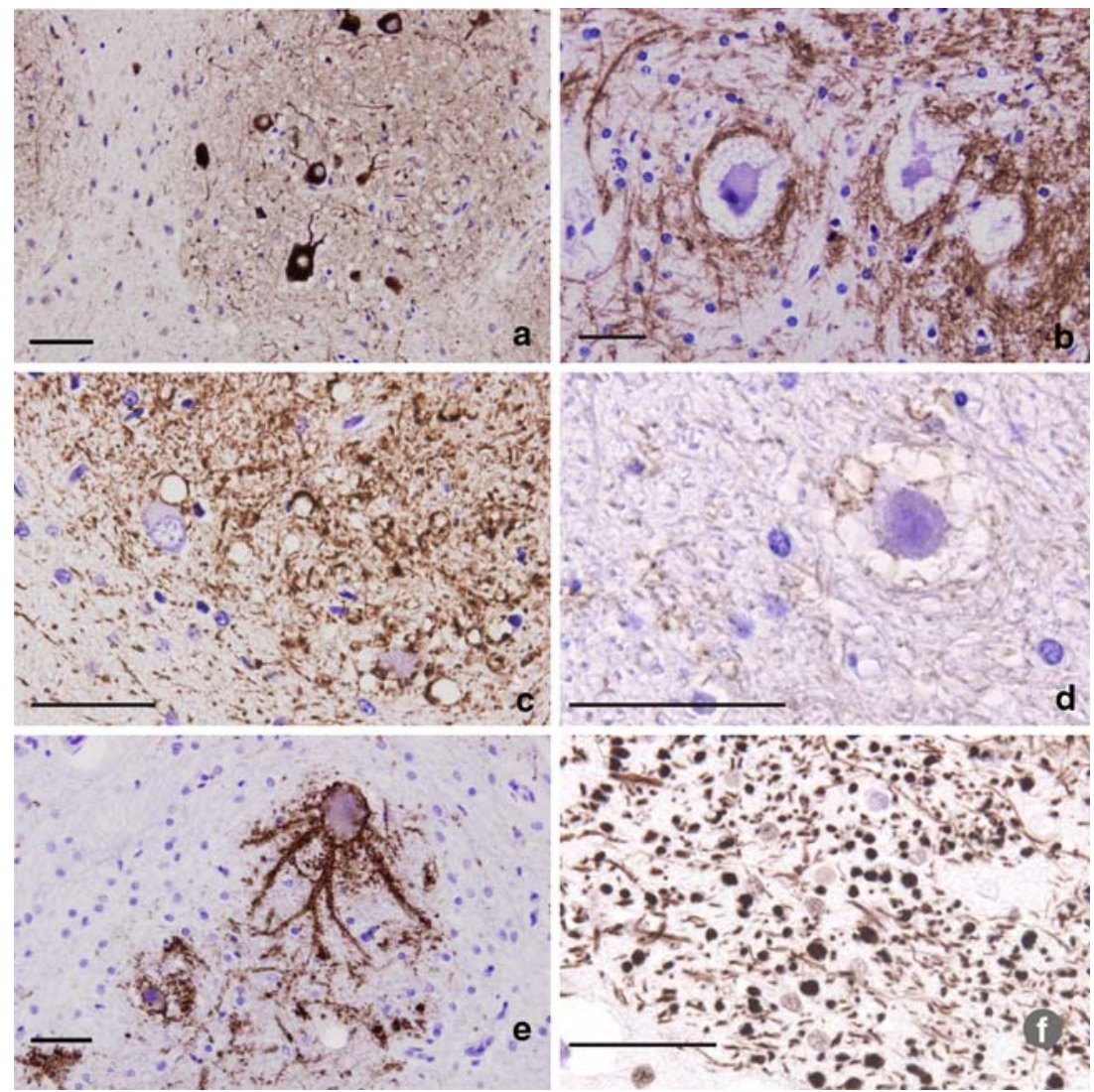

development appears normal on routine staining. A diffuse increase of reactive astroglia and activated microglia is seen throughout the neocortex and subcortical and central white matter in all cases. Except for postischemic changes in one case (6), the hippocampal and dentate gyri are normal.

\section{Olivopontocerebellar hypoplasia}

Case 7: this male baby was born at 38 weeks ga. The amount of amniotic fluid was normal. Spontaneous respiration was absent. He had trismus, severe tremor/clonus and varus deformity of the legs. On autopsy, the supratentorial parts of the brain are small size, but of normal appearance, while the cerebellum and brainstem are disproportionately small.

The cerebellum is remarkable for its complete sparing of the nodulus and flocculus, its severe cortical depletion on the dorsal side and hypoplastic cortical development on the ventral side of the hemispheres (Fig. 12). Folial development of the neocerebellum is severely restricted with few folia on its dorsal parts. Microscopic examination of the flocculus and vermis is normal. Neocerebellar hemispheric cortex shows isomorphic gliosis through transformed Bergmann cells (Fig. 12b). MAP-2 staining shows some retained neuronal elements (Fig. 12c, d). The dentate nuclei are broken up into small islands that can only be retrieved by neuron-specific staining. This reveals a few small clusters with well-developed dentate neurons left in place (Fig. 12e, f). The inferior olivary nuclei have the form of horseshoes without folding, and with the segments of the nucleus missing on both sides (Fig. 13). The accessory olivary nuclei are spared. The ventral pons is severely underdeveloped with most of the pontine nuclei missing, dense gliosis and absence of CD68 staining. A diffuse astrocytic gliosis is found throughout the cerebral hemispheres, especially affecting the subcortical white matter and the fiber tracts of the striatum. The neocortex is unremarkable.

\section{Unfolded protein response}

We previously observed morphological changes in the endoplasmic reticulum (ER) in a biopsy of a $\mathrm{PCH}-2$ patient by electron microscopy. This led us to investigate whether the unfolded protein response (UPR) - a stress response of the ER [40]-is activated in neurons of $\mathrm{PCH}-2$ patients. The UPR was studied in sections from the frontal cortex (5/ 6 patients) and the cerebellar hemispheres, which included the dentate nucleus (6/6 patients). As a marker of induction of the UPR, we used an antibody specific for the phosphorylated pancreatic ER kinase (pPERK), which is an ER stress transducer that is only phosphorylated during the UPR. Previously, we have used this method to show UPR 
Fig. 9 Cerebellar cyst, case 5. a Vimentin, c HLA-DR. a Collapsed cyst seamed by astroglial reaction. b T1-weighted axial image of posterior fossa of patient at 1 month age shows the cysts in expanded state. c Macrophage reaction on the inside of the cyst
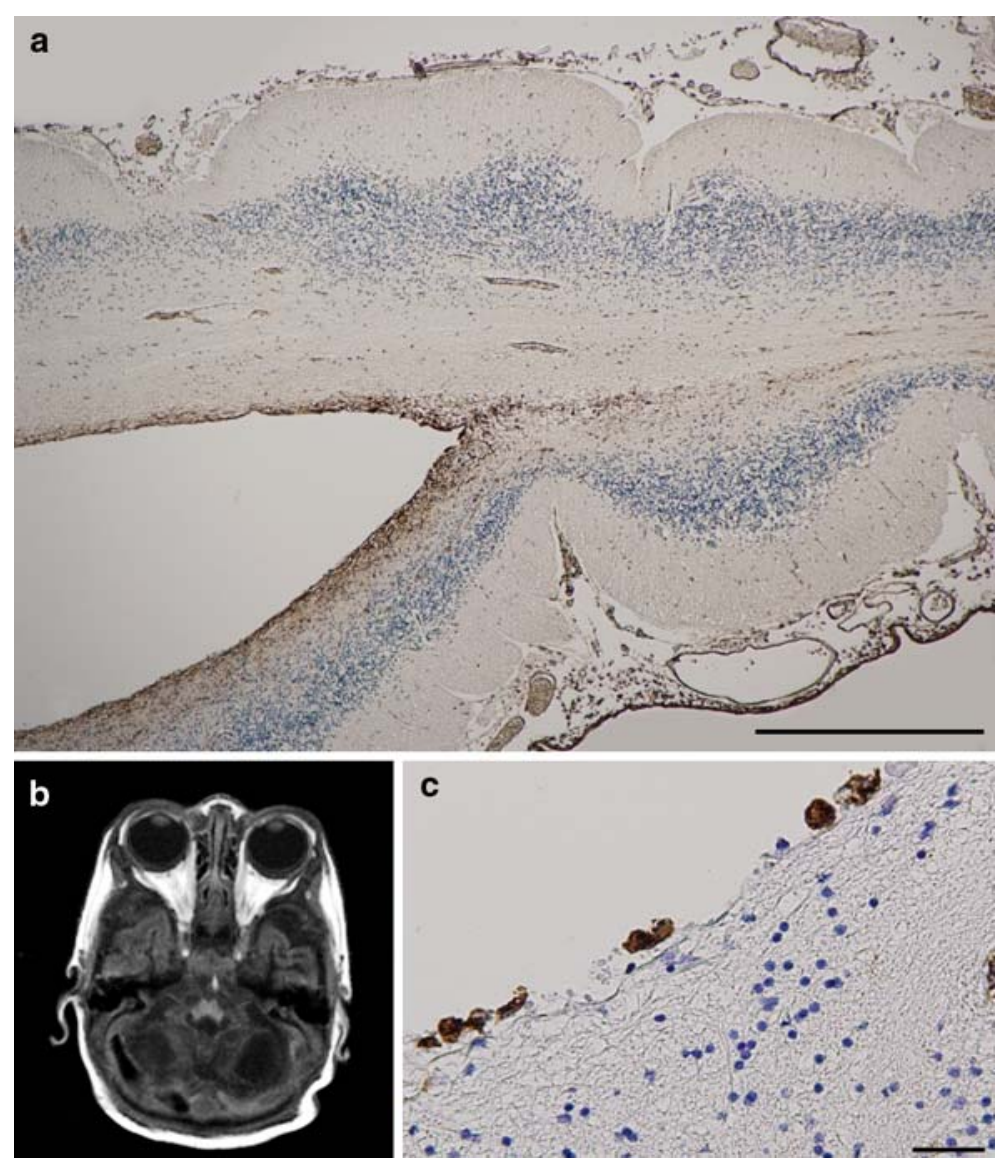

activation in the brain of patients with Alzheimer's disease [19] or Parkinson's disease [20]. We find no immunopositive staining for pPERK in any of the patients, neither in the cortex, nor in the cerebellum. Eukaryotic initiation factor $2 \alpha($ IF $2 \alpha)$ is the substrate of pPERK and using a peIF $2 \alpha$ antibody, increased staining intensity is observed as compared to the control patient in the Purkinje cells of patients 2,4 and 5. This is not found in patient 1 and severe degeneration in patients 3 and 6 precludes proper analysis. Apart from the fact that differences in intensities between pathological samples are not always readily interpretable, this does not provide evidence for UPR activation in the absence of pPERK reactivity, because other kinases can also phosphorylate eIF2 $\alpha$. The ER chaperone BiP is upregulated during the UPR, but in accordance with the pPERK data, no obvious differences in levels are observed. In summary, we find no evidence for activation of the UPR.

\section{Discussion}

Cerebellar cortex, dentate nucleus and olivary complex

The series of six PCH-2 cases presents developmental and regressive abnormalities of the cerebellar cortex, dentate nuclei and olivary nuclei. Cerebellar cortical findings range between short unbranched folia in the least-developed cerebellum (case 5) to shortened folia with a decreased number of branches. In the most affected cerebella, poorly developed folia alternate with flat stretches of surface that lack proper cortical lining. A similar observation is reported in PCH-1 cases [13, 14, 46], OPCH [35], a case classified as "neocerebellar hypoplasia with combined olivo-pontodentatal degeneration" [24], and PCH-2 with spasticity [42]. Reactive glial changes were reported in PCH-1 [46]. All "empty" segments of cortex in the present study have a similar appearance: a single evenly spaced row of transformed Bergmann cells, positively staining for vimentin. Synaptophysin and MAP-2 staining reveal the sparse presence of nerve fibers. This is the first time this patten is observed by immunohistochemistry. The earliest stages of cerebellar development are probably normal because they require the coordinate development and juxtaposition of two cell types: the Purkinje cells that arise by radial outward migration and the external granule cells that arise by tangential migration and enter the future cortex to become internal granule cells [39]. The microscopic structure of the "empty" segments betrays previous cortical development. The earliest estimate for the loss of cortex in these segments follows from the afolial stretches in patients 5 and 6 


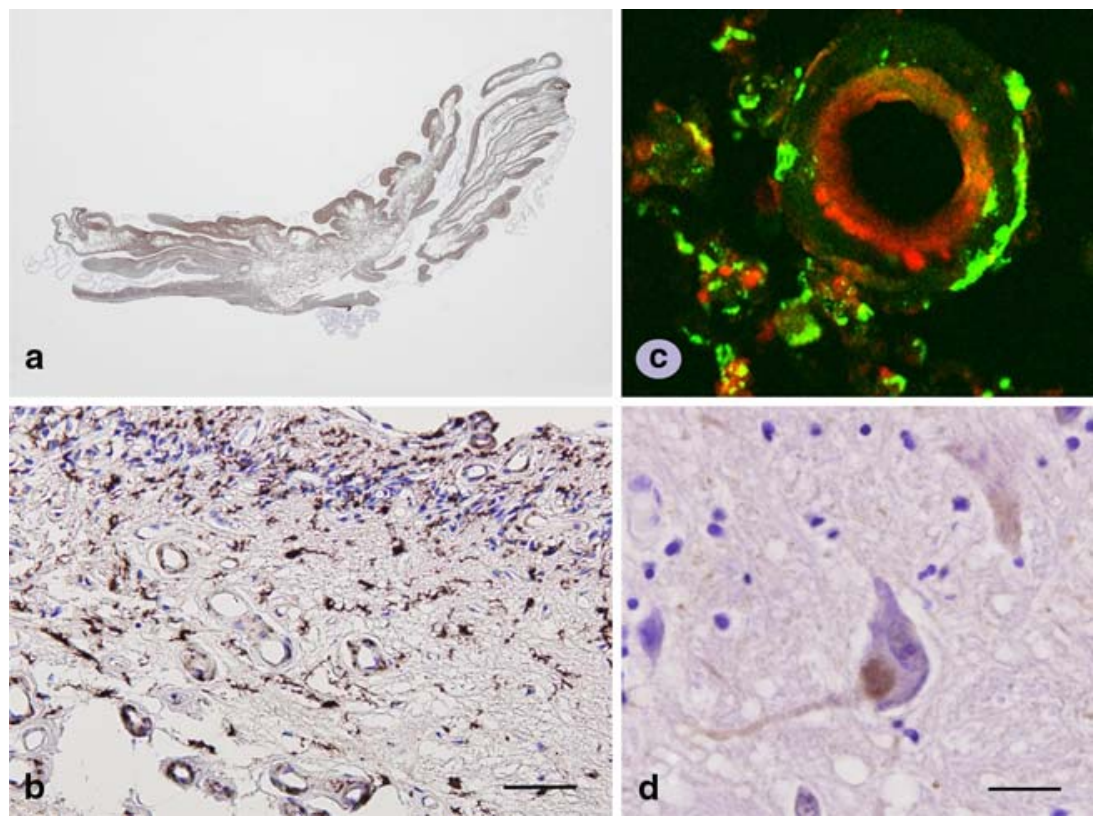

Fig. 10 Regressive changes, case 3. Stains: a GFAP, b HLA-DR, c double labeling for HLA-DR ( reen) and ubiquitine (red), confocal laser microscopy, $\mathbf{d}$ ubiquitin. a Transverse cerebellar section with cortical and subcortical gliosis, loss of the subcortical and central cerebellar structure, in part cystic, broken cortical lining in places suggest segmental lesions similar to other cases before ultimate decay; b abundance of reactive macrophages, predominantly in and surrounding blood vessels with thickened vessel walls; $\mathbf{c}$ ubiquitin is mostly restricted to vessel wall and macrophages are partly positive for ubiquitin; d neuron of nucleus magnus raphes (pontine tegmentum) with cytoplasmic inclusion and positivity extending into the neurite. Marker bars: $\mathbf{b}=200 \mu \mathrm{m}, \mathbf{c}=50 \mu \mathrm{m}$
Fig. 11 Inferior olivary nucleus. Stains: a-c MAP-2, d vimentin. case 6 and 5: segmental loss of inferior olivary nucleus with intact dorsal (a) and medial (b) accessory nuclei. c Case 3. Complete loss of nucleus, including accessory nuclei, only sparse isolated neurons remaining, indicated by arrowheads; inset shows magnified solitary olivary neuron. d Case 3. Outline of lost lateral leaf reveals mature folding. Marker bars $=0.5 \mathrm{~mm}$
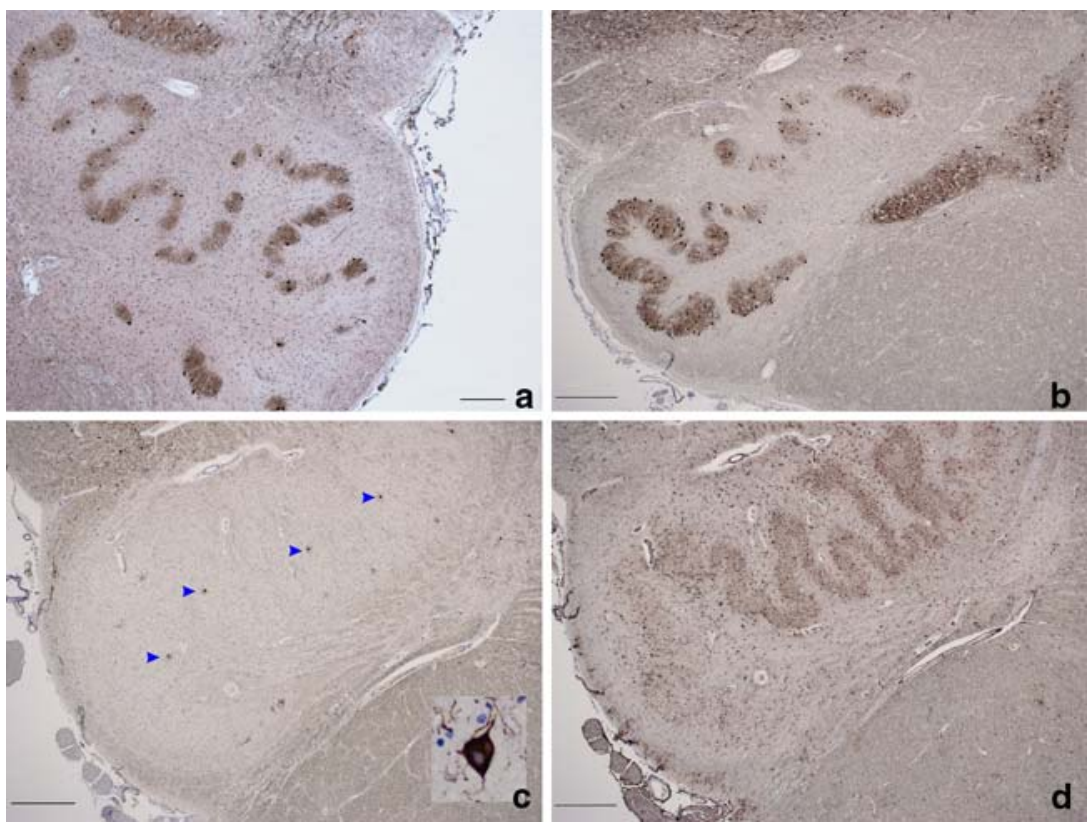

that represent a stage of cerebellar development prior to or coinciding with beginning folial outgrowth, around 25 weeks gestational age in the human fetus [39]. Cortical segmental loss, however, is not restricted to afolial segments, but is also seen in more developed hemispheric cortex (e.g., Fig. 5h, i) and mature vermis. This suggests a time frame for segmental loss of cerebellar cortex from incipient foliation to encompass late cortical development. It cannot be established presently whether segmental loss is a completed process or still active at the time of demise. No microscopic vascular changes are seen in the vicinity of segmental lesions and their distribution is not restricted to arterial boundary zones. Also, cerebellar vascular changes observed in one case (3) are not found in any of the other 
Fig. 12 OPCH, case 7. Stains: a, e synaptophysin, b vimentin, c, d, f MAP-2. a Whole mount transverse section of cerebellum and medulla oblongata: denuded hemispheric cerebellar cortex and striking preservation of nodulus and flocculi. b Hemispheric cortex lined by a single row of fibrous astrocytes. c "Empty" cerebellar cortex, arrows indicate some remaining neurons. d Higher magnification of c, arrowhead indicates bipolar neuron, possibly basket cell; arrow indicates malpositioned Purkinje cell. e Overview of cerebellar hemisphere, arrows at residues of dentate nucleus. f Single residual island of dentate nucleus with welldeveloped neurons. Marker bars: b $200 \mathrm{~m} \mu$, c $1 \mathrm{~mm}$, d $100 \mathrm{~m} \mu$, e $1 \mathrm{~mm}, \mathbf{f} 100 \mathrm{~m} \mu$

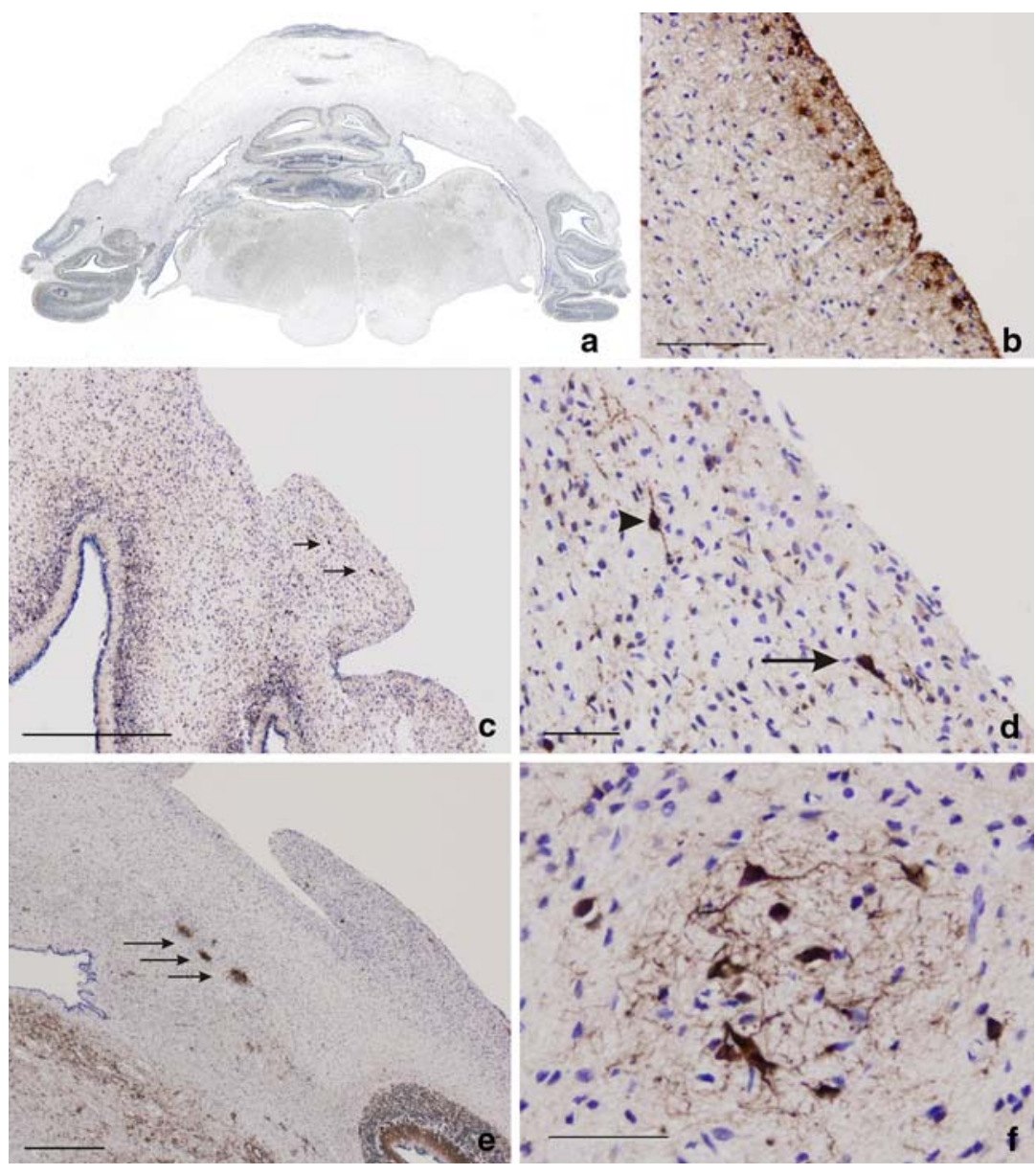

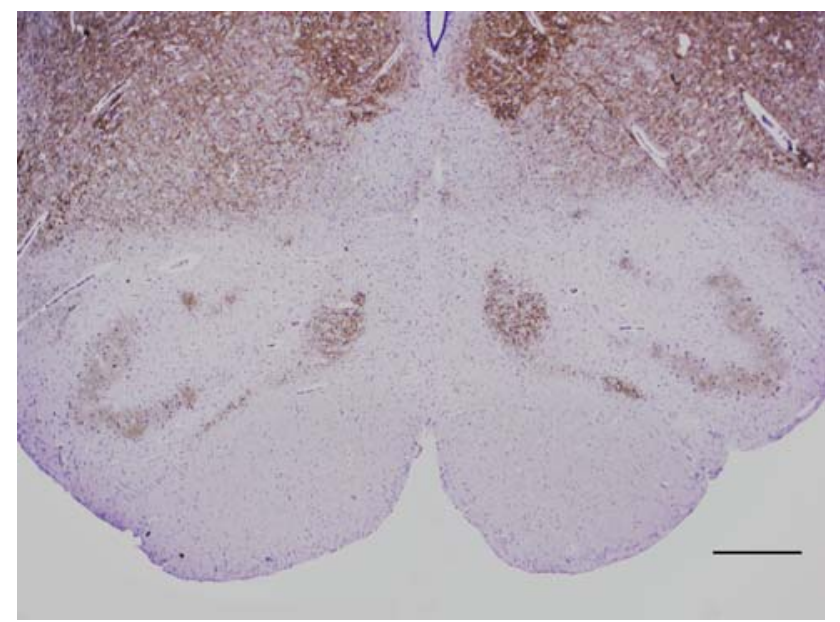

Fig. $13 \mathrm{OPCH}$, case 7. Stain, synaptophysin. Medulla oblongata with olivary nuclei. Inferior olivary nucleus with complete lack of folding. Lateral and medial leaves with segmental defects. Medial accessory olive is preserved in its dorsal part and shows discontinuities in its ventral part. Marker bar $0.5 \mathrm{~mm}$

cases and therefore offer no explanation for segmental cortical lesions. The spectrum of changes in the inferior olivary nucleus in this series similarly encompasses early and late developments. In five out of six cases, the degree of folding is immature, while in the sixth case (case 3), a mature folding pattern of the nucleus can be gauged by observing the outline of the astroglial scar. According to one study in human fetal material [30], the mature form of the nucleus is reached with a $300 \mathrm{~mm}$ crown-heel length, i.e., 22-27 weeks. Segmental loss is observed in 5/6 cases in the olivary nucleus, and the sequence of progressive loss may have been similar in the remaining oldest case (case 3) with loss of the entire nucleus. Segmental lesions in the inferior olivary nucleus in PCH were reported in 1926 [26]. The consistency of this finding is shown in the present series using immunohistochemistry. The third structure with a segmental pattern of loss is the dentate nucleus. We have no evidence in this series of a normal outline of the dentate nucleus during any phase of its development. One previous report [32] however relates a glial scar linking the "islands" of the dentate in $\mathrm{PCH}$.

We examined a case classified as OPCH and on comparison to the PCH-2 series found important similarities: (1) flat stretches of cerebellar cortex and ghosts of primitive folia carrying microscopic remnants of a pre-existent developed cortex, (2) dentate islands with well-developed neurons, (3) segmental loss in the inferior olivary nucleus, (4) sparing of nodulus and flocculus. These findings reveal a 
remarkable analogy to the segmental cortical lesions in PCH-2. Differences between PCH-2 and OPCH are: (1) the horseshoe appearance of the inferior olivary nucleus compared to the diminished folding in $\mathrm{PCH}-2$ indicates earlier onset of the pathological process in $\mathrm{OPCH}$, (2) the extent of afolial cerebellar cortex.

\section{Regressive changes}

In addition to developmental abnormalities, some findings indicate an ongoing process, such as cytoplasmic ubiquitin positivity in pontine tegmental neurons in case 3 , the widespread presence of astrocytic and microglial reaction and the abundance of lipopigment, e.g., in cerebral cortical, cerebellar cortical, dentate and olivary neurons. Cyst formation in the cerebellar white matter (cases 3,5) and vascular changes in the cerebellum (case 3 ) also signify a regressive element in $\mathrm{PCH}-2$. A previously made observation on darkened stretches of endoplasmic reticulum in cortical neurons in a cortical biopsy [5] prompted us to investigate the ER stress response with negative results.

\section{Comparison of $\mathrm{PCH}-2$ to other neurodegenerative disorders}

The nearest equivalent in neuropathological terms to $\mathrm{PCH}-$ 2 is the group previously named olivo-ponto-cerebellar atrophy (OPCA), presently known as spinocerebellar ataxia (SCA). While olivary and/or dentate nucleus may be affected, none of the typical features, especially dentate fragmentation, segmental lesions of the cerebellar cortex and olivary nucleus is part of the pathological spectrum of these disorders $[8,25]$. Pathological studies on autosomal recessive or X-linked central nervous system disorders with onset in childhood and predominant impact on the cerebellum include the Høyeraal-Hreidarsson syndrome [1, 22], progressive encephalopathy, hypsarrhythmia, optic atrophy (PEHO) [16], primary granular cell degeneration of the cerebellum [31], and infantile onset spinocerebellar ataxia with neuropathy (IOSCA) [27]. These disorders display cerebellar atrophy rather than hypoplasia, lack the segmental cortical anomalies, the characteristic fragmentation of the dentate and the segmental loss of the olivary nucleus described above with the possible exception of IOSCA in which segmental loss of the olivary nucleus is documented [27]. Neurometabolic disorders of glycosylation, especially congenital disorder of glycosylation (CDG) type 1A and the alpha-dystroglycanopathies manifest cerebellar hypoplasia, often in combination with pontine hypoplasia. Neuropathological findings in CDG1A do not include circumscript dentate, olivary or cerebellar cortical defects [2, 21]. Alphadystroglycanopathies combine a severe type of congenital muscular dystrophy with cerebral, cerebellar and brainstem malformation and deficiency of alpha-dystroglycan (alpha
DG) [29]. Alpha DG deficiency in this group probably causes a tangential neuronal migration defect affecting migration from the rhombic lip into the cerebellum and ventral pons. The resulting pathology [17] causes heterotopia and impaired foliation in the cerebellum. Summarizing previous and present data on $\mathrm{PCH}-2$, this disorder bears a number of features not encountered in other disorders with the exception of $\mathrm{PCH}-1$, which appears to be closely related but for the involvement of spinal anterior horn cells in the latter.

\section{Neural mechanisms}

A question may be raised whether segmental lesions in the three nuclear systems, as observed in PCH-2, could be effected through a single mechanism. Cerebellar cortex, olivary nuclei and dentate nuclei are functionally linked through the climbing fiber system, which is topographically organized in sagittal modules each formed by a segment of the olivary nuclear complex, its collaterals to the cerebellar nuclei, including the dentate nucleus, and a strip of cerebellar cortex [45]. A sagittal parcelleation of the cerebellum in biochemically distinct regions, however develops independent of the olivo-cerebellar projection [43]. Lesions of the olivary complex are not known to cause segmental lesions in other parts of the climbing fiber system. Experimental administration of the drugs harmaline or ibogaine to rats results in tremor and loss of sagittal strips of vermis. The role of the olivary nucleus in this type of segmental damage is highlighted by prior chemical ablation of the inferior olivary nucleus by 3 -acetyl pyridine, which prevents the segmental damage from occurring. Overexcitation of the olivary nucleus rather than primary loss is apparently required for this type of lesion to occur [34]. The type of lesion reported in rodents is reminescent of the segmental lesions in PCH-2. One argument in support of a possible role of the olivo-cerebellar projection in $\mathrm{PCH}-2$ is the selectivity of the cerebellar cortex involved, in which the vermis and flocculus are relatively spared. The climbing fiber output of the inferior olivary nucleus to the cerebellum is mainly directed to the cerebellar hemispheres. The flocculus and nodulus, both relatively spared in $\mathrm{PCH}-2$ and in $\mathrm{OPCH}$, are reciprocally connected to the vestibular nuclei [45]. We have not found abnormalities in the vestibular nuclei in our series.

In addition to cortical segmental loss, a more diffuse kind of degeneration is found, which includes loss of cortical elements such as Purkinje cells and granule cells. This lends a "moth-eaten" aspect to the myelinated nerve bundles in the cores of folia and central cerebellar white matter, similar to the more common cerebellar degenerations such as in spino-cerebellar ataxias. Some findings such as gliosis in the central parts of the cerebellum and axonal swelling 
suggest an ongoing process. Study of the cell somata of the nucleus dentatus reveals some peculiarities in all patients where the nucleus could be identified, not seen in controls, such as perisomatic "vacuolation" that was even seen on routine HE staining. The presence of calbindin in some of these "vacuoles" suggests swollen nerve endings.

\section{Genetics and epigenetics}

Varying degrees of hypoplastic folial development are found in three patients $(4,5,6)$ who share the same ancestors (Fig. 3c-h). This indicates that modifying factors bear on the expression of this genetic condition. Patient 3, the patient with the longest survival, who died at 22 years, differed from other patients in having vascular anomalies in the cerebellum. This phenomenon may be caused by an unknown age-bound complication of the disease. However, a genotype distinct from the others cannot be excluded pending the unraveling of the genetic basis of $\mathrm{PCH}-2$.

Acknowledgment The authors would like to thank Prof. J.M. Rozemuller, Department of Neuropathology, Free University Medical Centre for the helpful discussions and Mrs. L.M.E. Stoets, Department of Clinical Genetics, University of Amsterdam for the genealogical studies.

\section{References}

1. Aalfs CM, van den Berg H, Barth PG, Hennekam RCM (1995) The Høyeraal-Hreidarsson syndrome: the fourth case of a separate entity with prenatal growth retardation, progressive pancytopenia and cerebellar hypoplasia. Eur J Pediatr 154:304-308

2. Aronica E, van Kempen AA, van der Heide M, Poll-The BT, van Slooten HJ, Troost D, Rozemuller-Kwakkel JM (2005) Congenital disorder of glycosylation type Ia: a clinicopathological report of a newborn infant with cerebellar pathology. Acta Neuropathol (Berl) 109:433-442

3. Albrecht S, Schneider MC, Belmont J, Armstrong DL (1993) Fatal infantile encephalopathy with olivopontocerebellar hypoplasia and micrencephaly. Acta Neuropathol 85:394-399

4. Arts WFM, Hofstee Y, Drejer GF, Beverstock GC, Oosterwijk JC (1995) Cerebellar and brainstem hypoplasia in a child with a partial monosomy for the short arm of chromosome 5 and partial trisomy for the short arm of chromosome 10 . Neuropediatrics $26: 41-$ 44

5. Barth PG, Vrensen GFJM, Uylings HBM, Oorthuys JWE, Stam FC (1990) Inherited syndrome of microcephaly, dyskinesia and pontocerebellar hypoplasia: a systemic atrophy with early onset. J Neurol Sci 97:25-42

6. Barth PG (1993) Pontocerebellar hypoplasias. An overview of a group of inherited neurodegenerative disorders with fetal onset. Brain Dev 15:411-422

7. Barth PG, Blennow G, Lenard H-G, Begeer JH, van der Kley JM, Hanefeld F, Peters ACB, Valk J (1995) The syndrome of autosomal recessive pontocerebellar hypoplasia, microcephaly and extrapyramidal dyskinesia (pontocerebellar hypoplasia type 2): compiled data from ten pedigrees. Neurology 45:311-317

8. Berciano J (1982) Olivopontocerebellar atrophy. A review of 117 cases. J Neurol Sci 53:253-272
9. Biemond A (1955) Hypoplasia ponto-neocerebellaris, with malformation of the dentate nucleus. Folia Psychiatr Neurol (Amst) $58: 2-7$

10. Brouwer B (1924) Hypoplasia ponto-neocerebellaris. Psychiatr Neurol (Amst) 6:461-473

11. Chaves-Vischer V, Pizzolato GP, Hanquinet S, Maret A, Bottani A, Haenggeli CA (2000) Early fatal pontocerebellar hypoplasia in premature twin sisters. Eur J Paediatr Neurol 4:171-176

12. Dekaban AS, Sadowsky D (1978) Changes in brain weights during the span of human life: relation of brain weights to body heights and body weights. Ann Neurol 4:345-6

13. De León GA, Grover WE, D’Cruz CA (1984) Amyotrophic cerebellar hypoplasia: a specific form of infantile spinal atrophy. Acta Neuropathol (Berl) 63:282-286

14. Goutières F, Aicardi J, Farkas E (1977) Anterior horn cell disease associated with pontocerebellar hypoplasia in infants. J Neurol Neurosurg Psychiatr 40:370-378

15. Guihard-Costa AM, Larroche J-C (1990) Differential growth between the fetal brain and its infratentorial part. Early Hum Dev 23:27-40

16. Haltia M, Somer M (1993) Infantile cerebello-optic atrophy. Neuropathology of the progressive encephalopathy syndrome with edema, hypsarrhythmia and optic atrophy (the PEHO syndrome). Acta Neuropathol 85:241-247

17. Haltia M, Leivo I, Somer H, Pihko H, Paetau A, Kivela T, Tarkkanen A, Tome F, Engvall E, Santavuori P (1997) Muscle-eye-brain disease: a neuropathological study. Ann Neurol 41:173-178

18. Hashimoto K, Takeuchi Y, Kida Y, Hasegawa H, Kantake M, Sasaki A, Asanuma K, Isumi H, Takashima S (1998) Three siblings of fatal infantile encephalopathy with olivopontocerebellar hypoplasia and microcephaly. Brain Dev 20:169-174

19. Hoozemans JJ, Veerhuis R, Rozemuller AJ, Baas F, Eikelenboom $\mathrm{P}$, Scheper W (2005) The unfolded protein response is activated in Alzheimer's disease. Acta Neuropathol (Berl) 110:165-172

20. Hoozemans JJ, van Haastert ES, Eikelenboom P, de Vos RA, Rozemuller JM, Scheper W (2007) Activation of the unfolded protein response in Parkinson's disease. Biochem Biophys Res Commun 354:707-711

21. Horslen SP, Clayton PT, Harding BN, Hall NA, Keir G, Winchester B (1991) Olivopontocerebellar atrophy of neonatal onset and disialotransferrin developmental deficiency syndrome. Arch Dis Child 66:1027-1032

22. Høyeraal HM, Lamvik J, Moe PJ (1970) Congenital hypoplastic thrombocytopenia and cerebral malformations in two brothers. Acta Paediatr Scand 59:185-191

23. Jaeken J, Carchon H (1993) The carbohydrate-deficient glycoprotein syndromes - an overview. J Inherit Metab Dis 16:813-820

24. Kawagoe T, Jacob H (1986) Neocerebellar hypoplasia with systemic combined olivo-ponto-dentatal degeneration in a 9-day-old baby: contribution to the problem of relations between malformation and systemic degeneration in early life. Clin Neuropathol 5:203-208

25. Koeppen AH (2005) The pathogenesis of spinocerebellar ataxia. Cerebellum 4:62-73

26. Koster S (1926) Two cases of hypoplasia ponto-neocerebellaris. Acta Psychiatr (Københ) 47-76

27. Lönnqvist T, Paetau A, Nikali K, von Boguslawski K, Pihko H (1998) Infantile onset spinocerebellar ataxia with sensory neuropathy (IOSCA): neuropathological features. J Neurol Sci 161:5765

28. Messerschmidt A, Brugger PC, Boltshauser E, Zoder G, Sterniste W, Birnbacher R, Prayer D (2005) Disruption of cerebellar development: potential complication of extreme prematurity. AJNR Am J Neuroradiol 26:1659-1667

29. Moore SA, Saito F, Chen J, Michele DE, Henry MD, Messing A, Cohn RD, Ross-Barta SE, Westra S, Williamson RA, Hoshi T, 
Campbell KP (2002) Deletion of brain dystroglycan recapitulates aspects of congenital muscular dystrophy. Nature 418:422-425

30. Murofushi K (1974) Normalentwicklung und Dysgenesien von Dentatum und Oliva inferior. Acta Neuropathol (Berl) 27:317-328

31. Norman RM (1940) Primary degeneration of the granular layer of the cerebellum: an unusual form of familial cerebellar atrophy occurring in early life. Brain 63:365-379

32. Norman RM, Urich HC (1958) Cerebellar hypoplasia associated with systemic degeneration in early life. J Neurol Neurosurg Psychiatr 21:159-166

33. Norman RM (1961) Cerebellar hypoplasia in Werdnig-Hoffmann disease. Arch Dis Child 36:96-101

34. O'Hearn E, Molliver ME (1993) Degeneration of Purkinje cells in parasagittal zones of the cerebellar vermis after treatment with ibogaine or harmaline. Neuroscience 55:303-10

35. Park SH, Becker-Catania S, Gatti RA, Crandall BF, Emelin JK, Vinters HV (1998) Congenital olivopontocerebellar atrophy - report of two siblings with paleo- and neocerebellar atrophy. Acta Neuropathologica 96:315-321

36. Patel MS, Becker LE, Toi A, Armstrong DL, Chitayat D (2006) Severe, fetal-onset form of olivopontocerebellar hypoplasia in three sibs: PCH type 5? Am J Med Genet A 140:594-603

37. Peiffer J, Pfeiffer RA (1977) Hypoplasia ponto-neocerebellaris. J Neurol 215:241-51

38. Rajab A, Mochida GH, Hill A, Ganesh V, Bodell A, Riaz A, Grant PE, Shugart YY, Walsh CA (2003) A novel form of pontocerebel- lar hypoplasia maps to chromosome 7q11-21. Neurology 60:1664-1667

39. Rakic C, Sidman RL (1970) Histogenesis of cortical layers in human cerebellum, particularly the lamina dissecans. J Comp Neur 139:473-500

40. Rutkowski DT, Kaufman RJ (2004) A trip to the ER: coping with stress. Trends Cell Biol 14:20-28

41. Simonati A (1997) Pontocerebellar hypoplasia with dystonia: clinico-pathological findings in a sporadic case. Childs Nerv Syst 13:642-647

42. Schneider H, Zetune R, Kirchschläger H (1973) Ponto-neocerebelläre hypoplasie bei zwei geschwistern. In: Jellinger K (ed) Current topics in neuropathology. Selected papers, 4th Danube Symposium on Neuropathology, Vienna Fakultas Verlag, Vienna pp 109-114

43. Sotelo C (2004) Cellular and genetic regulation of the development of the cerebellar system. Prog Neurobiol 72:295-339

44. Steinlin M, Klein A, Haas-Lude K, Zafeiriou D, Strozzi S, Muller T,Gubser-Mercati D, Schmitt Mechelke T, Krageloh-Mann I, Boltshauser E (2007) Pontocerebellar hypoplasia type 2: Variability in clinical and imaging findings. Eur J Paediatr Neurol 11:146152

45. Voogd J, Glickstein M (1998) The anatomy of the cerebellum. Trends Neurosci 21:370-375

46. Weinberg AG, Kirkpatrick JB (1975) Cerebellar hypoplasia in Werdnig-Hoffmann disease. Dev Med Child Neurol 17:511-516 